\title{
Valuative invariants for polymatroids
}

\author{
Harm Derksen $\|^{\|}$and Alex Fink ${ }^{2}$ \\ ${ }^{1}$ University of Michigan \\ ${ }^{2}$ University of California, Berkeley
}

\begin{abstract}
Many important invariants for matroids and polymatroids, such as the Tutte polynomial, the Billera-JiaReiner quasi-symmetric function, and the invariant $\mathcal{G}$ introduced by the first author, are valuative. In this paper we construct the $\mathbb{Z}$-modules of all $\mathbb{Z}$-valued valuative functions for labeled matroids and polymatroids on a fixed ground set, and their unlabeled counterparts, the $\mathbb{Z}$-modules of valuative invariants. We give explicit bases for these modules and for their dual modules generated by indicator functions of polytopes, and explicit formulas for their ranks. Our results confirm a conjecture of the first author that $\mathcal{G}$ is universal for valuative invariants.

Résumé. Beaucoup des invariants importants des matroïdes et polymatroïdes, tels que le polynôme de Tutte, la fonction quasi-symmetrique de Billera-Jia-Reiner, et l'invariant $\mathcal{G}$ introduit par le premier auteur, sont valuatifs. Dans cet article nous construisons les $\mathbb{Z}$-modules de fonctions valuatives aux valeurs entières des matroïdes et polymatrö̈des étiquetés définis sur un ensemble fixe, et leurs équivalents pas étiquetés, les $\mathbb{Z}$-modules des invariants valuatifs. Nous fournissons des bases des ces modules et leurs modules duels, engendrés par fonctions charactéristiques des polytopes, et des formules explicites donnants leurs rangs. Nos résultats confirment une conjecture du premier auteur, que $\mathcal{G}$ soit universel pour les invariants valuatifs.
\end{abstract}

Keywords: polymatroids, polymatroid polytopes, decompositions, valuations

\section{Introduction}

Matroids were introduced by Whitney in 1935 (see [22]) as a combinatorial abstraction of linear dependence of vectors in a vector space. Some standard references are [21] and [17]. Polymatroids are multiset analogs of matroids and appeared in the late 1960s (see [9, 13]). There are many distinct but equivalent definitions of matroids and polymatroids, for example in terms of bases, independent sets, flats, polytopes or rank functions. For polymatroids, the equivalence between the various definitions is given in [13]. We will stick to the definition in terms of rank functions:

Definition 1.1 Suppose that $X$ is a finite set (the ground set) and $\mathrm{rk}: 2^{X} \rightarrow \mathbb{N}=\{0,1,2, \ldots\}$, where $2^{X}$ is the set of subsets of $X$. Then $(X, \mathrm{rk})$ is called a polymatroid if:

1. $\operatorname{rk}(\emptyset)=0$;

2. $\mathrm{rk}$ is weakly increasing: if $A \subseteq B$ then $\operatorname{rk}(A) \leq \operatorname{rk}(B)$;

\footnotetext{
${ }^{\dagger}$ Supported by NSF grants DMS 0349019 and DMS 0901298.

() The full version of this paper is arXiv:0908.2988.

1365-8050 @ 2010 Discrete Mathematics and Theoretical Computer Science (DMTCS), Nancy, France
} 
3. rk is submodular: $\operatorname{rk}(A \cup B)+\operatorname{rk}(A \cap B) \leq \operatorname{rk}(A)+\operatorname{rk}(B)$ for all $A, B \subseteq X$.

If moreover $\mathrm{rk}(\{x\}) \leq 1$ for every $x \in X$, then $(X, \mathrm{rk})$ is called a matroid.

An isomorphism $\varphi:\left(X, \operatorname{rk}_{X}\right) \rightarrow\left(Y, \operatorname{rk}_{Y}\right)$ is a bijection $\varphi: X \rightarrow Y$ such that $\operatorname{rk}_{Y} \circ \varphi=\operatorname{rk}_{X}$. Every polymatroid is isomorphic to a polymatroid with ground set $\underline{d}=\{1,2, \ldots, d\}$ for some nonnegative integer $d$. The rank of a polymatroid $(X, \mathrm{rk})$ is $\operatorname{rk}(X)$.

Our matroid notations will receive the subscript ${ }_{M}$, and our polymatroid notations the subscript $\mathrm{PM}_{\mathrm{M}}$. We will write $(\mathrm{P}) \mathrm{M}(d, r)$ when we want to refer to both in parallel.

Let $S_{(\mathrm{P}) \mathrm{M}}(d, r)$ be the set of all (poly)matroids with ground set $\underline{d}$ of rank $r$, A function $f$ on $S_{(\mathrm{P}) \mathrm{M}}(d, r)$ is a (poly)matroid invariant if $f((\underline{d}, \mathrm{rk}))=f\left(\left(\underline{d}, \mathrm{rk}^{\prime}\right)\right)$ whenever $(\underline{d}, \mathrm{rk})$ and $\left(\underline{d}, \mathrm{rk}^{\prime}\right)$ are isomorphic. Let $S_{(\mathrm{P}) \mathrm{M}}^{\mathrm{sym}}(d, r)$ be the set of isomorphism classes in $S_{(\mathrm{P}) \mathrm{M}}(d, r)$. Invariant functions on $S_{(\mathrm{P}) \mathrm{M}}(d, r)$ correspond to functions on $S_{(\mathrm{P}) \mathrm{M}}^{\mathrm{sym}}(d, r)$. Let $Z_{(\mathrm{P}) \mathrm{M}}(d, r)$ and $Z_{(\mathrm{P}) \mathrm{M}}^{\mathrm{sym}}(d, r)$ be the $\mathbb{Z}$-modules freely generated by the symbols $\langle\mathrm{rk}\rangle$ for rk in $S_{(\mathrm{P}) \mathrm{M}}(d, r)$ and $S_{(\mathrm{P}) \mathrm{M}}^{\mathrm{sym}}(d, r)$ respectively. For an abelian group $A$, every function $f: S_{(\mathrm{P}) \mathrm{M}}^{(\mathrm{sym})}(d, r) \rightarrow A$ extends uniquely to a group homomorphism $Z_{(\mathrm{P}) \mathrm{M}}^{(\mathrm{sym})}(d, r) \rightarrow A$.

To a (poly)matroid $(\underline{d}, \mathrm{rk})$ one can associate its base polytope $Q(\mathrm{rk})$ in $\mathbb{R}^{d}$ (see Definition 2.2). For $d \geq 1$, the dimension of this polytope is $\leq d-1$. The indicator function of a polytope $\Pi \subseteq \mathbb{R}^{d}$ is denoted by $[\Pi]: \mathbb{R}^{d} \rightarrow \mathbb{Z}$. Let $P_{(\mathrm{P}) \mathrm{M}}(d, r)$ be the $\mathbb{Z}$-module generated by all $[Q(\mathrm{rk})]$ with $(\underline{d}, \mathrm{rk}) \in S_{(\mathrm{P}) \mathrm{M}}(d, r)$. We also define an analogue $P_{\mathrm{P}(\mathrm{M})}^{\mathrm{sym}}(d, r)$ by a certain pushout (see Section 6 ).

Definition 1.2 Suppose that $A$ is an abelian group. A function $f: S_{(\mathrm{P}) \mathrm{M}}(d, r) \rightarrow A$ is strongly valuative if there exists a group homomorphism $\widehat{f}: P_{(\mathrm{P}) \mathrm{M}}(d, r) \rightarrow A$ such that for all $(\underline{d}, \mathrm{rk}) \in S_{(\mathrm{P}) \mathrm{M}}(d, r)$,

$$
f((\underline{d}, \mathrm{rk}))=\widehat{f}([Q(\mathrm{rk})]) .
$$

Many interesting functions on matroids are valuative. Among these is the Tutte polynomial, one of the most important matroid invariants [5, 7]. Other valuative functions on matroids include the quasisymmetric function $\mathcal{F}$ for matroids of Billera, Jia and Reiner introduced in [3], and the first author's quasi-symmetric function $\mathcal{G}$ introduced in [8]. Speyer's invariant defined in [19] using $K$-theory is strictly speaking not valuative, but its composition with a certain automorphism of $Z_{* \mathrm{M}}^{\mathrm{sym}}(d, r)$ is valuative. Valuative invariants and additive invariants can be useful for deciding whether a given matroid polytope has a decomposition into smaller matroid polytopes (see the discussion in [3, Section 7]). Matroid polytope decompositions appeared in the work of Lafforgue $([14,15])$ on compactifications of a fine Schubert cell in the Grassmannian associated to a matroid.

It follows from Definition 1.2 that the dual $P_{(\mathrm{P}) \mathrm{M}}(d, r)^{\vee}=\operatorname{Hom}_{\mathbb{Z}}\left(P_{(\mathrm{P}) \mathrm{M}}(d, r), \mathbb{Z}\right)$ is the space of all $\mathbb{Z}$-valued valuative functions on $S_{(\mathrm{P}) \mathrm{M}}(d, r)$. Likewise $P_{(\mathrm{P}) \mathrm{M}}^{\mathrm{sym}}(d, r)^{\vee}$ is the space of all $\mathbb{Z}$-valued valuative invariants. Let $p_{(\mathrm{P}) \mathrm{M}}^{(\mathrm{sym})}(d, r)$ be the rank of $P_{(\mathrm{P}) \mathrm{M}}^{(\mathrm{sym})}(d, r)$.

We will give explicit bases for each of the spaces $P_{(\mathrm{P}) \mathrm{M}}(d, r)$ and $P_{(\mathrm{P}) \mathrm{M}}^{\mathrm{sym}}(d, r)$ and their duals (see Theorems 5.16.1. Corollaries 5.3 6.2. From these we obtain the following formulas:

\section{Theorem 1.3}

a. $p_{\mathrm{M}}^{\mathrm{sym}}(d, r)=\left(\begin{array}{l}d \\ r\end{array}\right)$ and $\sum_{0 \leq r \leq d} p_{\mathrm{M}}^{\mathrm{sym}}(d, r) x^{d-r} y^{r}=\frac{1}{1-x-y}$. 

b. $p_{\mathrm{PM}}^{\mathrm{sym}}(d, r)=\left\{\begin{array}{ll}\left(\begin{array}{c}r+d-1 \\ r\end{array}\right) & \text { if } d \geq 1 \text { or } r \geq 1 ; \\ 1 & \text { if } d=r=0\end{array}\right.$ and $\sum_{r=0}^{\infty} \sum_{d=0}^{\infty} p_{\mathrm{PM}}^{\mathrm{sym}}(d, r) x^{d} y^{r}=\frac{1-x}{1-x-y}$
c. $\sum_{0 \leq r \leq d} \frac{p_{\mathrm{M}}(d, r)}{d !} x^{d-r} y^{r}=\frac{x-y}{x e^{-x}-y e^{-y}}$.
d. $p_{\mathrm{PM}}(d, r)=\left\{\begin{array}{ll}(r+1)^{d}-r^{d} & \text { if } d \geq 1 \text { or } r \geq 1 ; \\ 1 & \text { if } d=r=0,\end{array} \quad\right.$ and $\sum_{d=0}^{\infty} \sum_{r=0}^{\infty} \frac{p_{\mathrm{PM}}(d, r) x^{d} y^{r}}{d !}=\frac{e^{x}(1-y)}{1-y e^{x}}$.

In particular, the following theorem, which is a corollary of Corollary 6.3 , proves a conjecture of the first author in [8]:

Theorem 1.4 The invariant $\mathcal{G}$ is universal for all valuative (poly)matroid invariants, i.e., the coefficients of $\mathcal{G}$ span the vector space of all valuative (poly)matroid invariants with values in $\mathbb{Q}$.

Definition 1.5 Suppose that $d>0$. A valuative function $f: S_{(\mathrm{P}) \mathrm{M}}(d, r) \rightarrow A$ is said to be additive, if $f((\underline{d}, \mathrm{rk}))=0$ whenever the dimension of $Q(\mathrm{rk})$ is $<d-1$.

In Sections 8 and 9 we construct bigraded modules $T_{(\mathrm{P}) \mathrm{M}}$ and $T_{(\mathrm{P}) \mathrm{M}}^{\mathrm{sym}}$ such that $T_{(\mathrm{P}) \mathrm{M}}(d, r)^{\vee}$ is the space of all additive functions on $S_{(\mathrm{P}) \mathrm{M}}(d, r)$ and $T_{(\mathrm{P}) \mathrm{M}}^{\mathrm{sym}}(d, r)^{\vee}$ is the space of all additive invariants. Let $t_{(\mathrm{P}) \mathrm{M}}(d, r)$ be the rank of $T_{(P) M}(d, r)$ and $t_{(P) M}^{\mathrm{sym}}(d, r)$ be the rank of $T_{(P) M}^{\mathrm{sym}}(d, r)$. Then we have the following formulas:

\section{Theorem 1.6}
a. $\prod_{0 \leq r \leq d}\left(1-x^{d-r} y^{r}\right)^{t_{\mathrm{M}}^{\mathrm{sym}}(d, r)}=1-x-y$.
b. $\prod_{r, d}\left(1-x^{d} y^{r}\right)^{t_{\mathrm{PM}}^{\mathrm{sym}}(d, r)}=\frac{1-x-y}{1-y}$.
c. $\sum_{r, d} \frac{t_{\mathrm{M}}(d, r)}{d !} x^{d-r} y^{r}=\log \left(\frac{x-y}{x e^{-x}-y e^{-y}}\right)$.
d. $t_{\mathrm{PM}}(d, r)=\left\{\begin{array}{ll}r^{d-1} & \text { if } d \geq 1 \\ 0 & \text { if } d=0,\end{array}\right.$ and $\sum_{r, d} \frac{t_{\mathrm{PM}}(d, r)}{d !} x^{d} y^{r}=\log \left(\frac{e^{x}(1-y)}{1-y e^{x}}\right)$.

\section{Polymatroids and their polytopes}

By a polyhedron we will mean a finite intersection of closed half-spaces. A polytope is a bounded polyhedron. It is convenient to have a polyhedral analogue of polymatroid polytopes, so we make the following definition.

Definition 2.1 A function $2^{X} \rightarrow \mathbb{Z} \cup\{\infty\}$ is called a megamatroid if it has the following properties:

1. $\operatorname{rk}(\emptyset)=0$;

2. $\operatorname{rk}(X) \in \mathbb{Z}$; 
3. $\operatorname{rk}$ is submodular: if $\operatorname{rk}(A), \operatorname{rk}(B) \in \mathbb{Z}$, then $\operatorname{rk}(A \cup B), \operatorname{rk}(A \cap B) \in \mathbb{Z}$ and $\operatorname{rk}(A \cup B)+\operatorname{rk}(A \cap B) \leq$ $\operatorname{rk}(A)+\operatorname{rk}(B)$.

Obviously, every matroid is a polymatroid, and every polymatroid is a megamatroid. The rank of a megamatroid $(X, \mathrm{rk})$ is the integer $\operatorname{rk}(X)$. We will use notations for megamatroids analogous to our notations for (poly)matroids but with the subscript $\mathrm{MM}$. We will use the subscript ${ }_{* \mathrm{M}}$ and say "*matroid" when we want to refer to megamatroids or polymatroids or matroids in parallel.

Definition 2.2 For a megamatroid ( $\underline{d}, \mathrm{rk})$, we define its base polyhedron $Q(\mathrm{rk})$ as the set of all $\left(y_{1}, \ldots, y_{d}\right) \in$ $\mathbb{R}^{d}$ such that $y_{1}+y_{2}+\cdots+y_{d}=\operatorname{rk}(X)$ and $\sum_{i \in A} y_{i} \leq \operatorname{rk}(A)$ for all $A \subseteq X$.

If $\mathrm{rk}$ is a polymatroid then $Q(\mathrm{rk})$ is a polytope, called the base polytope of rk. In fact $Q(\mathrm{rk})$ is always nonempty. The base polytope of a polymatroid $(\underline{d}, \mathrm{rk})$ of rank $r$ is contained in the simplex

$$
\Delta_{\mathrm{PM}}(d, r)=\left\{\left(y_{1}, \ldots, y_{d}\right) \in \mathbb{R}^{d} \mid y_{1}, \ldots, y_{d} \geq 0, y_{1}+y_{2}+\cdots+y_{d}=r\right\}
$$

and the base polytope of a matroid $(\underline{d}$, rk) of rank $r$ is contained in the hypersimplex

$$
\Delta_{\mathrm{M}}(d, r)=\left\{\left(y_{1}, \ldots, y_{d}\right) \in \mathbb{R}^{d} \mid 0 \leq y_{1}, \ldots, y_{d} \leq 1, y_{1}+y_{2}+\cdots+y_{d}=r\right\} .
$$

The next theorem generalises a theorem of Gelfand-Goresky-MacPherson-Serganova [10] on matroids.

Theorem 2.3 A convex polyhedron contained in $y_{1}+\cdots+y_{d}=r$ equals $Q(M)$ for some megamatroid $M$ if and only if for every face $F$ of $\Pi$, the linear hull $\operatorname{lhull}(F)$ is of the form $z+W$ where $z \in \mathbb{Z}^{d}$ and $W$ is spanned by vectors of the form $e_{i}-e_{j}$.

Polymatroid polyhedra are, up to translation, the lattice polytopes among the generalized permutohedra of [18] or the submodular rank tests of [16].

\section{The valuative property}

There are essentially two definitions of the valuative property in the literature, which we will refer to as the strong valuative and the weak valuative properties. The equivalence of these definitions is shown in [12] and [20] when valuations are defined on sets of polyhedra closed under intersection. We show their equivalence for megamatroid polytopes, which are not such a set.

Definition 3.1 A megamatroid polyhedron decomposition is a decomposition $\Pi=\Pi_{1} \cup \Pi_{2} \cup \cdots \cup \Pi_{k}$ such that $\Pi, \Pi_{1}, \ldots, \Pi_{k}$ are megamatroid polyhedra, and $\Pi_{i} \cap \Pi_{j}$ is empty or contained in a proper face of $\Pi_{i}$ and of $\Pi_{j}$ for all $i \neq j$.

A megamatroid polyhedron decomposition $\Pi=\Pi_{1} \cup \cdots \cup \Pi_{k}$ is a (poly)matroid polytope decomposition if $\Pi, \Pi_{1}, \ldots, \Pi_{k}$ are (poly)matroid polytopes.

For a megamatroid polyhedron decomposition $\Pi=\Pi_{1} \cup \Pi_{2} \cup \cdots \cup \Pi_{k}$ we define $\Pi_{I}=\bigcap_{i \in I} \Pi_{i}$ if $I \subseteq\{1,2, \ldots, k\}$, and $\Pi_{\emptyset}=\Pi$. Define

$$
m_{\mathrm{val}}\left(\Pi ; \Pi_{1}, \ldots, \Pi_{k}\right)=\sum_{I \subseteq\{1,2, \ldots, k\}}(-1)^{|I|} m_{I} \in Z_{\mathrm{MM}}(d, r),
$$

where $m_{I}=\left\langle\mathrm{rk}^{I}\right\rangle$ if $\mathrm{rk}^{I}$ is the megamatroid with $Q\left(\mathrm{rk}^{I}\right)=\Pi_{I}$, and $m_{I}=0$ if $\Pi_{I}=\emptyset$. 
Definition 3.2 A homomorphism of abelian groups $f: Z_{* \mathrm{M}}(d, r) \rightarrow A$ is called weakly valuative if for every megamatroid polyhedron decomposition $\Pi=\Pi_{1} \cup \Pi_{2} \cup \cdots \cup \Pi_{k}$ we have

$$
f\left(m_{\mathrm{val}}\left(\Pi ; \Pi_{1}, \ldots, \Pi_{k}\right)\right)=0 .
$$

For a polyhedron $\Pi$ in $\mathbb{R}^{d}$, let $[\Pi]$ denote its indicator function. Define $P_{\mathrm{MM}}(d, r)$ as the $\mathbb{Z}$-module generated by all $[Q(\mathrm{rk})]$, where rk lies in $S_{\mathrm{MM}}(d, r)$. There is a natural $\mathbb{Z}$-module homomorphism

$$
\Psi_{* \mathrm{M}}: Z_{* \mathrm{M}}(d, r) \rightarrow P_{* \mathrm{M}}(d, r)
$$

such that $\Psi_{* \mathrm{M}}(\langle\mathrm{rk}\rangle)=[Q(\mathrm{rk})]$ for all $\mathrm{rk} \in S_{* \mathrm{M}}(d, r)$.

Definition 3.3 A homomorphism of groups $f: Z_{* \mathrm{M}}(d, r) \rightarrow A$ is strongly valuative if there exists a group homomorphism $\widehat{f}: P_{* \mathrm{M}}(d, r) \rightarrow$ A such that $f=\widehat{f} \circ \Psi_{* \mathrm{M}}$.

The map $\Psi_{* \mathrm{M}}$ has the weak valuative property, which shows that the strong valuative property implies the weak valuative property. In fact the two valuative properties are equivalent, and in view of this we may speak of the valuative property.

Theorem 3.4 A homomorphism $f: Z_{* \mathrm{M}}(d, r) \rightarrow$ A of abelian groups is weakly valuative if and only if it is strongly valuative.

\section{Decompositions into cones}

A chain of length $k=$ : length $(\underline{X})$ in $\underline{d}$ is $\underline{X}: \emptyset \subset X_{1} \subset \cdots \subset X_{k-1} \subset X_{k}=\underline{d}$ (here $\subset$ denotes proper inclusion). If $d>0$ then every chain has length $\geq 1$, but for $d=0$ there is exactly one chain, namely $\emptyset=$ $\underline{0}$, and this chain has length 0 . For a chain $\underline{X}$ of length $k$ and a $k$-tuple $\underline{r}=\left(r_{1}, r_{2}, \ldots, r_{k}\right) \in(\mathbb{Z} \cup\{\infty\})^{k}$, we define a megamatroid polyhedron

$$
R_{\mathrm{MM}}(\underline{X}, \underline{r})=\left\{\left(y_{1}, \ldots, y_{d}\right) \in \mathbb{R}^{d} \mid \sum_{i=1}^{d} y_{i}=r_{k}, \forall j \sum_{i \in X_{j}} y_{i} \leq r_{j}\right\} .
$$

We will always use the conventions $r_{0}=0, X_{0}=\emptyset$. Note that the polytopes $R_{\mathrm{MM}}(\underline{X}, \underline{r})$ are fulldimensional cones in $\left\{\sum_{y=1}^{d} y_{i}=r\right\}$.

The next theorem is an analogue of the Brianchon-Gram Theorem [4, 11] for megamatroid polytopes.

Theorem 4.1 For any megamatroid $\mathrm{rk}: 2^{\underline{d}} \rightarrow \mathbb{Z} \cup\{\infty\}$ we have

$$
[Q(\mathrm{rk})]=\sum_{\underline{X}}(-1)^{d-\operatorname{length}(\underline{X})}\left[R_{\mathrm{MM}}\left(\underline{X},\left(\operatorname{rk}\left(X_{1}\right), \ldots, \operatorname{rk}\left(X_{k}\right)\right)\right)\right] .
$$

Example 4.2 Consider the case where $d=3$ and $r=3$, and $\operatorname{rk}$ is defined by $\operatorname{rk}(\{1\})=\operatorname{rk}(\{2\})=$ $\operatorname{rk}(\{3\})=2, \operatorname{rk}(\{1,2\})=\operatorname{rk}(\{2,3\})=\operatorname{rk}\{(1,3\})=3, \operatorname{rk}(\{1,2,3\})=4$. The right of Figure 1 depicts the decomposition using the Brianchon-Gram theorem of a polytope $Q_{\varepsilon}(\mathrm{rk})$, which is defined by a certain perturbation of the inequalities defining $Q(\mathrm{rk})$. Note how the summands in the decomposition correspond to the faces of $Q_{\varepsilon}(\mathrm{rk})$. In the limit where the perturbation approaches $0, Q_{\varepsilon}(\mathrm{rk})$ tends to $Q(\mathrm{rk})$ and we obtain the left of Figure 1 This is exactly the decomposition in Theorem 4.1. In this decomposition, the summands do not correspond bijectively to the faces of $Q(\mathrm{rk})$.

The dashed triangle is the triangle defined by $y_{1}, y_{2}, y_{3} \geq 0, y_{1}+y_{2}+y_{3}=4$. 

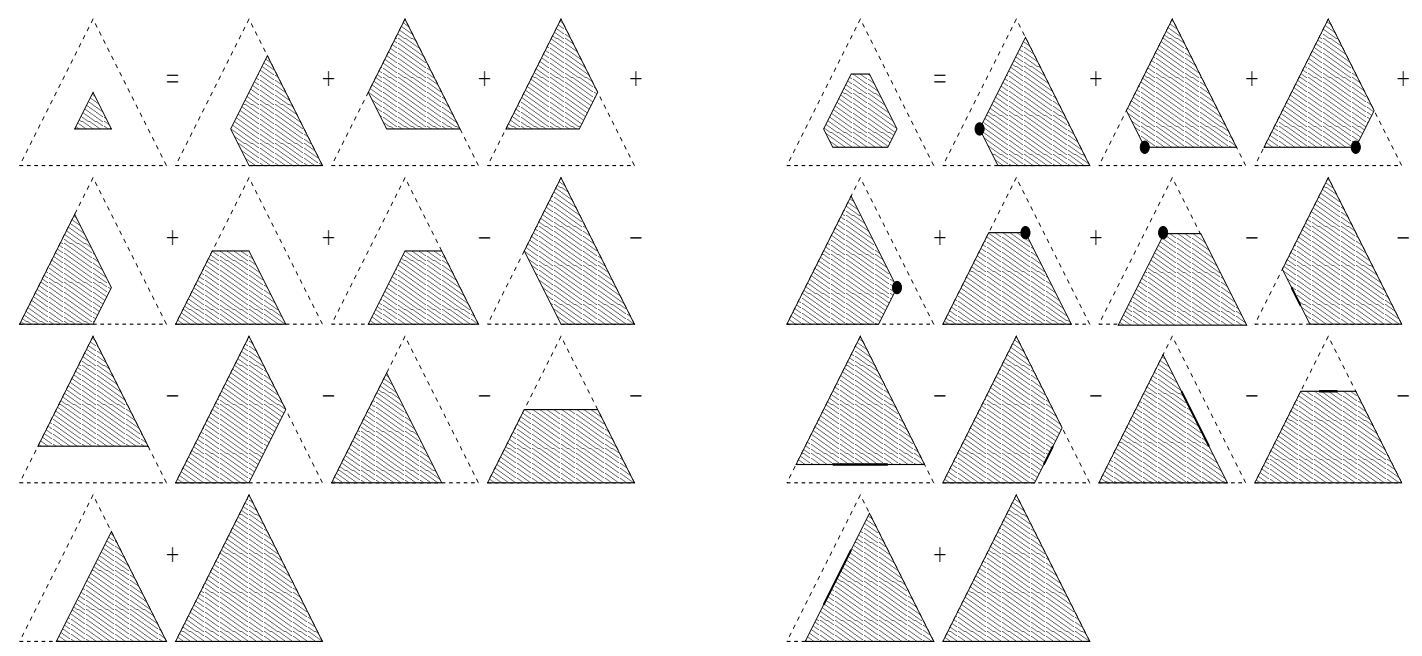

Fig. 1: At left, a decomposition of $Q(\mathrm{rk})$ as in Theorem 4.1 At right, a decomposition of a polytope $Q_{\epsilon}(\mathrm{rk})$.

\section{Valuations}

Suppose that $d \geq 1$. Let $\mathfrak{p}_{\mathrm{MM}}(d, r)$ be the set of all pairs $(\underline{X}, \underline{r})$ such that $\underline{X}$ is a chain of length $k$ $(1 \leq k \leq d)$ and $\underline{r}=\left(r_{1}, r_{2}, \ldots, r_{k}\right)$ is an integer vector with $r_{k}=r$. We define $R_{(\mathrm{P}) \mathrm{M}}(\underline{X}, \underline{r})=$ $R_{\mathrm{MM}}(\underline{X}, \underline{r}) \cap \Delta_{(\mathrm{P}) \mathrm{M}}(d, r)$. If $R_{(\mathrm{P}) \mathrm{M}}(\underline{X}, \underline{r})$ is nonempty, then it is a (poly)matroid base polytope. Define $\mathfrak{p}_{\mathrm{PM}}(d, r) \subseteq \mathfrak{p}_{\mathrm{MM}}(d, r)$ as the set of all pairs $(\underline{X}, \underline{r})$ with $0 \leq r_{1}<\cdots<r_{k}=r$. Let $\mathfrak{p}_{\mathrm{M}}(d, r)$ denote the set of all pairs $(\underline{X}, \underline{r}) \in \mathfrak{p}_{\mathrm{MM}}(d, r)$ such that $\underline{r}=\left(r_{1}, \ldots, r_{k}\right)$ for some $k(1 \leq k \leq d)$,

$$
0 \leq r_{1}<r_{2}<\cdots<r_{k}=r
$$

and

$$
0<\left|X_{1}\right|-r_{1}<\left|X_{2}\right|-r_{2}<\cdots<\left|X_{k-1}\right|-r_{k-1} \leq\left|X_{k}\right|-r_{k}=d-r .
$$

For $d=0$, we define $\mathfrak{p}_{\mathrm{MM}}(0, r)=\mathfrak{p}_{\mathrm{PM}}(0, r)=\mathfrak{p}_{\mathrm{M}}(0, r)=\emptyset$ for $r \neq 0$ and $\mathfrak{p}_{\mathrm{MM}}(0,0)=\mathfrak{p}_{\mathrm{PM}}(0,0)=$ $\mathfrak{p}_{\mathrm{M}}(0,0)=\{(\emptyset \subseteq \underline{0},())\}$.

Theorem 5.1 The group $P_{* \mathrm{M}}(d, r)$ is freely generated by the basis $\left\{\left[R_{* \mathrm{M}}(\underline{X}, \underline{r})\right] \mid(\underline{X}, \underline{r}) \in \mathfrak{p}_{* \mathrm{M}}(d, r)\right\}$.

Note that the basis of this theorem is a generating set by Theorem 4.1

Suppose that $\underline{X}$ is a chain of length $k$ and $\underline{r}=\left(r_{1}, \ldots, r_{k}\right)$ is an integer vector with $r_{k}=r$. Define a homomorphism $s_{\underline{X}, \underline{r}}: Z_{\mathrm{MM}}(d, r) \rightarrow \mathbb{Z}$ by

$$
s_{\underline{X}, \underline{r}}(\mathrm{rk})= \begin{cases}1 & \text { if } \operatorname{rk}\left(X_{j}\right)=r_{j} \text { for } j=1,2, \ldots, k, \\ 0 & \text { otherwise. }\end{cases}
$$

Proposition 5.2 The homomorphism $s_{\underline{X}, \underline{r}}$ is valuative.

Theorem 5.3 The group $P_{(\mathrm{P}) \mathrm{M}}(d, r)^{\vee}$ is freely generated by the basis $\left\{s_{\underline{X}, \underline{r}}:(\underline{X}, \underline{r}) \in \mathfrak{p}_{(\mathrm{P}) \mathrm{M}}(d, r)\right\}$.

If $\underline{X}$ is not a maximal chain, then $s_{\underline{X}, \underline{r}}$ is a linear combination of functions of the form $s_{\underline{X}^{\prime}, \underline{r}^{\prime}}$ where $\underline{X}^{\prime}$ is a maximal chain. The set of such functions $s_{\underline{X}^{\prime}, \underline{r}^{\prime}}$ appeared as the coordinates of the function $H$ defined in [1, §6], which was introduced there as a labeled analogue of $\mathcal{G}$. 


\section{Valuative invariants}

Let $Y_{* \mathrm{M}}(d, r)$ be the group generated by all $\langle\mathrm{rk}\rangle-\langle\mathrm{rk} \circ \sigma\rangle$ where rk $: 2^{\underline{d}} \rightarrow \mathbb{Z} \cup\{\infty\}$ is a $*$ matroid of rank $r$ and $\sigma$ is a permutation of $\underline{d}$. We define $Z_{* \mathrm{M}}^{\mathrm{sym}}(d, r)=Z_{* \mathrm{M}}(d, r) / Y_{* \mathrm{M}}(d, r)$. Let $\pi_{* \mathrm{M}}: Z_{* \mathrm{M}}(d, r) \rightarrow$ $Z_{* \mathrm{M}}^{\text {sym }}(d, r)$ be the quotient homomorphism. If $\mathrm{rk}_{X}: 2^{X} \rightarrow \mathbb{Z} \cup\{\infty\}$ is any $*$ matroid, then we can choose a bijection $\varphi: \underline{d} \rightarrow X$, where $d$ is the cardinality of $X$. Let $r=\operatorname{rk}_{X}(X)$. The image of $\left\langle\operatorname{rk}_{X} \circ \varphi\right\rangle$ in $Z_{* \mathrm{M}}^{\text {sym }}(d, r)$ does not depend on $\varphi$, and will be denoted by $\left[\mathrm{rk}_{X}\right]$. The $*$ matroids $\left(X, \mathrm{rk}_{X}\right)$ and $\left(Y, \mathrm{rk}_{Y}\right)$ are isomorphic if and only if $\left[\mathrm{rk}_{X}\right]=\left[\mathrm{rk}_{Y}\right]$. So we may think of $Z_{* \mathrm{M}}^{\mathrm{sym}}(d, r)$ as the free group generated by all isomorphism classes of rank $r *$ matroids on sets with $d$ elements.

Let $P_{(\mathrm{P}) \mathrm{M}}^{\mathrm{sym}}(d, r)$ be the pushout of the diagram

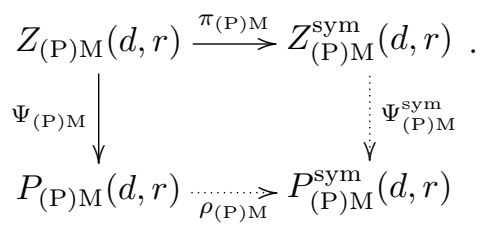

Then the dual space $P_{(\mathrm{P}) \mathrm{M}}^{\mathrm{sym}}(d, r)^{\vee}$ is exactly the set of all $\mathbb{Z}$-valued valuative (poly)matroid invariants. Define $\mathfrak{p}_{* \mathrm{M}}^{\mathrm{sym}}(d, r)$ as the set of all pairs $(\underline{X}, \underline{r}) \in \mathfrak{p}_{* \mathrm{M}}(d, r)$ such that every $X_{j}$ is of the form $\{1,2, \ldots, i\}$.

Theorem 6.1 The $\mathbb{Z}$-module $P_{\star \mathrm{M}}^{\mathrm{sym}}(d, r)$ is freely generated by all $\rho_{\star \mathrm{M}}\left(\left[R_{\star \mathrm{M}}(\underline{X}, \underline{r})\right]\right)$ with $(\underline{X}, \underline{r}) \in$ $\mathfrak{p}_{\star \mathrm{M}}^{\mathrm{sym}}(d, r)$.

The matroid polytopes $R_{\mathrm{M}}(\underline{X}, \underline{r})$ with $(\underline{X}, \underline{r}) \in \mathfrak{p}_{\star \mathrm{M}}^{\mathrm{sym}}(d, r)$ are the polytopes of Schubert matroids. Schubert matroids were first described by Crapo [6], and have since arisen in several contexts, prominent among these being the stratification of the Grassmannian into Schubert cells [2, §2.4].

For $(\underline{X}, \underline{r}) \in \mathfrak{p}_{\mathrm{MM}}^{\mathrm{sym}}(d, r)$, define a homomorphism $s_{\underline{X}, \underline{r}}^{\mathrm{sym}}: Z_{\mathrm{MM}}(d, r) \rightarrow \mathbb{Z}$ by

$$
s_{\underline{X}, \underline{r}}^{\mathrm{sym}}=\sum_{\sigma \underline{X}} s_{\sigma \underline{X}, \underline{r}}
$$

where the sum is over all chains $\sigma \underline{X}$ in the orbit of $\underline{X}$ under the action of the symmetric group.

Theorem 6.2 The $\mathbb{Q}$-vector space $P_{(\mathrm{P}) \mathrm{M}}^{\mathrm{sym}}(d, r)^{\vee} \otimes_{\mathbb{Z}} \mathbb{Q}$ of valuations $Z_{(\mathrm{P}) \mathrm{M}}^{\mathrm{sym}}(d, r) \rightarrow \mathbb{Q}$ has a basis given by the functions $s_{\underline{X}, \underline{r}}^{\mathrm{sym}}$ for $(\underline{X}, \underline{r}) \in \mathfrak{p}_{(\mathrm{P}) \mathrm{M}}^{\mathrm{sym}}(d, r)$.

For a sequence $\alpha=\left(\alpha_{1}, \ldots, \alpha_{d}\right)$ of nonnegative integers with $|\alpha|=\sum_{i} \alpha_{i}=r$, we define $u_{\alpha}=s_{\underline{X}, \underline{r}}: Z_{(\mathrm{P}) \mathrm{M}}^{\mathrm{sym}}(d, r) \rightarrow \mathbb{Z}$, where $\underline{X}_{i}=\underline{i}$ for $i=1,2, \ldots, r$ and $\underline{r}=\left(\alpha_{1}, \alpha_{1}+\alpha_{2}, \ldots, \alpha_{1}+\cdots+\alpha_{d}\right)$.

Corollary 6.3 The $\mathbb{Q}$-vector space $P_{\mathrm{PM}}^{\mathrm{sym}}(d, r)^{\vee} \otimes_{\mathbb{Z}} \mathbb{Q}$ of valuations $Z_{\mathrm{PM}}^{\mathrm{sym}}(d, r) \rightarrow \mathbb{Q}$ has a $\mathbb{Q}$-basis given by the functions $u_{\alpha}$, where $\alpha$ runs over all sequences $\left(\alpha_{1}, \ldots, \alpha_{d}\right)$ of nonnegative integers with $|\alpha|=r$.

Corollary 6.4 The $\mathbb{Q}$-vector space $P_{\mathrm{M}}^{\mathrm{sym}}(d, r)^{\vee} \otimes_{\mathbb{Z}} \mathbb{Q}$ of valuations $Z_{\mathrm{M}}^{\mathrm{sym}}(d, r) \rightarrow \mathbb{Q}$ has a $\mathbb{Q}$-basis given by all functions $u_{\alpha}$ where $\alpha$ runs over all sequences $\left(\alpha_{1}, \ldots, \alpha_{d}\right) \in\{0,1\}^{d}$ with $|\alpha|=r$. 


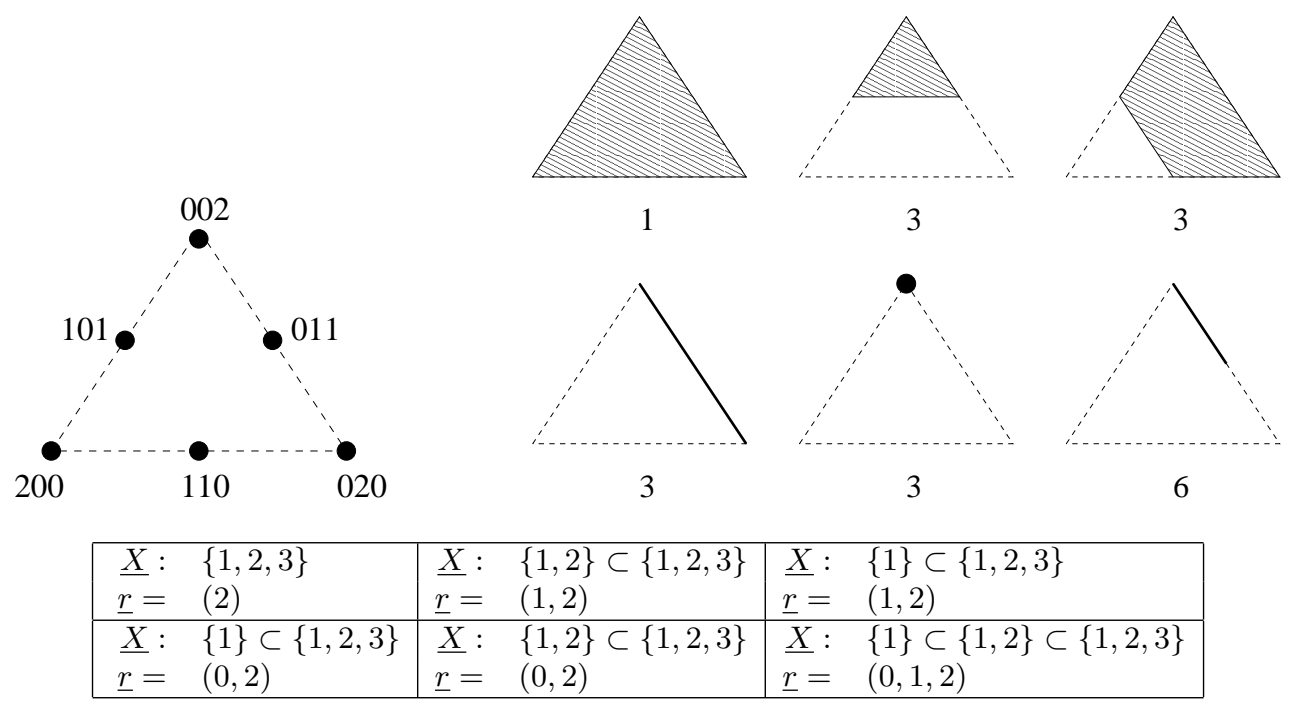

Fig. 2: A polymatroid example of Theorems 5.1 and 6.1

Example 6.5 Figures 2 and 3 are examples of both Theorems 5.1 and 6.1 for matroids in the cases $(d, r)=(3,2)$ and $(d, r)=(4,2)$, respectively. At top left are the polyhedra $\Delta_{(\mathrm{P}) \mathrm{M}}(d, r)$, containing all the (poly)matroid base polytopes. At top right are the polytopes $R(\underline{X}, \underline{r})$ for $(\underline{X}, \underline{r}) \in \mathfrak{p}_{(\mathrm{P}) \mathrm{M}}^{\mathrm{sym}}(d, r)$, and at bottom the corresponding pairs $(\underline{X}, \underline{r})$.

The symmetric group $\Sigma_{d}$ acts on $\Delta_{d}$ by permuting the coordinates. If $\Sigma_{d}$ acts on the generators $R(\underline{X}, \underline{r})$ with $(\underline{X}, \underline{r}) \in \mathfrak{p}_{(\mathrm{P}) \mathrm{M}}(d, r)$, then we get all $R(\underline{X}, \underline{r})$ with $(\underline{X}, \underline{r}) \in \mathfrak{p}_{(\mathrm{P}) \mathrm{M}}(d, r)$. In the figure, we have written under each polytope the cardinality of its $\Sigma_{d}$-orbit.

\section{Hopf algebra structures}

Define $Z_{* \mathrm{M}}=\bigoplus_{d, r} Z_{* \mathrm{M}}(d, r)$, and in a similar way define $Z_{* \mathrm{M}}^{\text {sym }}, P_{* \mathrm{M}}$, and $P_{* \mathrm{M}}^{\text {sym }}$. We can view $Z_{* \mathrm{M}}^{\text {sym }}$ as the $\mathbb{Z}$-module freely generated by all isomorphism classes of $*$ matroids. In this section we will only speak of the megamatroid objects; in every case, there are analogous matroid and polymatroid objects, which are substructures.

If $\mathrm{rk}_{1}: 2^{\underline{d}} \rightarrow \mathbb{Z} \cup\{\infty\}$ and $\mathrm{rk}_{2}: 2^{\underline{e}} \rightarrow \mathbb{Z} \cup\{\infty\}$ then we define $\mathrm{rk}_{1} \boxplus \mathrm{rk}_{2}: 2 \underline{\underline{d+e}} \rightarrow \mathbb{Z} \cup\{\infty\}$ by

$$
\left(\mathrm{rk}_{1} \boxplus \mathrm{rk}_{2}\right)(A)=\operatorname{rk}_{1}(A \cap \underline{d})+\operatorname{rk}_{2}(\{i \in \underline{e} \mid d+i \in A\})
$$

for any set $A \subseteq \underline{d+e}$. Note that $\boxplus$ is not commutative. We have a multiplication $\nabla: Z_{\mathrm{MM}} \otimes_{\mathbb{Z}} Z_{\mathrm{MM}} \rightarrow$ $Z_{\mathrm{MM}}$ defined by $\nabla\left(\left\langle\mathrm{rk}_{1}\right\rangle \otimes\left\langle\mathrm{rk}_{2}\right\rangle\right)=\left\langle\mathrm{rk}_{1} \boxplus \mathrm{rk}_{2}\right\rangle$, which makes $Z_{\mathrm{MM}}(d, r)$ into an associative (noncommutative) ring with 1 . The multiplication also respects the bigrading of $Z_{\mathrm{MM}}(d, r)$. The unit $\eta: \mathbb{Z} \rightarrow$ $Z_{\mathrm{MM}}(d, r)$ is given by $1 \mapsto\left\langle\mathrm{rk}_{0}\right\rangle$ where $\mathrm{rk}_{0}: 2^{\underline{0}} \rightarrow \mathbb{Z} \cup\{\infty\}$ is the unique megamatroid defined by $\operatorname{rk}(\emptyset)=0$.

Next, we define a comultiplication for $Z_{\mathrm{MM}}$. Suppose that $X=\left\{i_{1}, i_{2}, \ldots, i_{d}\right\}$ is a set of integers with $i_{1}<\cdots<i_{d}$ and rk $: 2^{X} \rightarrow \mathbb{Z} \cup\{\infty\}$ is a megamatroid. We define a megamatroid $\widehat{\mathrm{rk}}: 2^{\underline{d}} \rightarrow \mathbb{Z} \cup\{\infty\}$ 


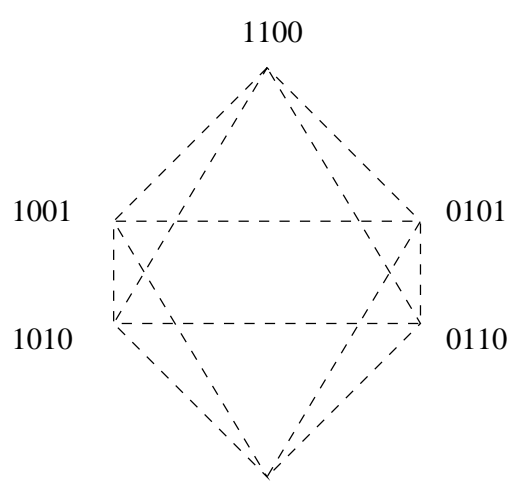

0011
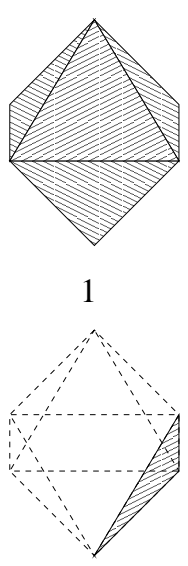

4
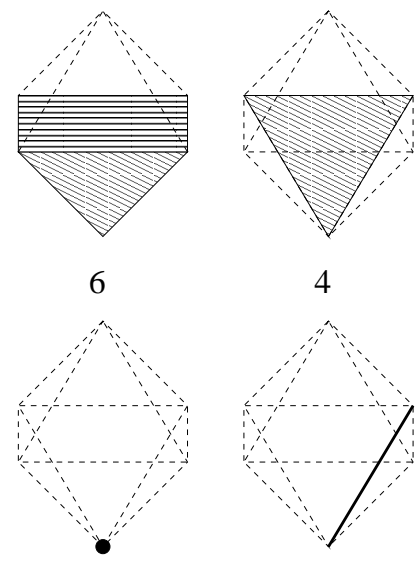

6

12

\begin{tabular}{|l|l|ll|}
\hline$\underline{X}:\{1,2,3,4\}$ & $\underline{X}:\{1,2\} \subset\{1,2,3,4\}$ & $\underline{X}:\{1,2,3\} \subset\{1,2,3,4\}$ \\
$\underline{r}=(2)$ & $\underline{r}=(1,2)$ & $\underline{r}=(1,2)$ \\
\hline$\underline{X}:\{1\} \subset\{1,2,3,4\}$ & $\underline{X}:\{1,2\} \subset\{1,2,3,4\}$ & $\underline{X}:\{1\} \subset\{1,2,3\} \subset\{1,2,3,4\}$ \\
$\underline{\underline{r}}=(0,2)$ & $\underline{\underline{r}}=(0,2)$ & $\underline{r}=(0,1,2)$ \\
\hline
\end{tabular}

Fig. 3: A matroid example of Theorems 5.1 and 6.1

by $\widehat{\operatorname{rk}}(A)=\operatorname{rk}\left(\left\{i_{j} \mid j \in A\right\}\right)$. If $\mathrm{rk}: 2^{X} \rightarrow \mathbb{Z} \cup\{\infty\}$ is a megamatroid and $B \subseteq A \subseteq X$ then we define $\mathrm{rk}_{A / B}: 2^{A \backslash B} \rightarrow \mathbb{Z} \cup\{\infty\}$ by $\operatorname{rk}_{A / B}(C)=\operatorname{rk}(B \cup C)-\operatorname{rk}(B)$ for all $C \subseteq A \backslash B$. We also define $\mathrm{rk}_{A}:=\mathrm{rk}_{A / \emptyset}$ and $\mathrm{rk}_{/ B}=\operatorname{rk}_{X / B}$.

We now define $\Delta: Z_{\mathrm{MM}} \rightarrow Z_{\mathrm{MM}} \otimes_{\mathbb{Z}} Z_{\mathrm{MM}}$ by

$$
\Delta(\langle\mathrm{rk}\rangle)=\sum_{A \subseteq \underline{d} ; \operatorname{rk}(A)<\infty}\left\langle\widehat{\mathrm{rk}_{A}}\right\rangle \otimes\left\langle\widehat{\mathrm{rk}_{/ A}}\right\rangle .
$$

where $A$ runs over all subsets of $\underline{d}$ for which $\operatorname{rk}(A)$ is finite. This comultiplication is coassociative, but not cocommutative. If rk $: 2^{\underline{d}} \rightarrow \mathbb{Z} \cup\{\infty\}$ is a megamatroid, then the counit is defined by $\epsilon(\langle\mathrm{rk}\rangle)=1$ (if $d=0$ ), 0 (otherwise). We omit here the definition of the antipode $S$.

It is well-known that $Z_{\mathrm{M}}^{\mathrm{sym}}$ has the structure of a Hopf algebra over $\mathbb{Z}$. In fact we have that $Z_{\mathrm{PM}}^{\mathrm{sym}}$ has a Hopf algebra structure, with $Z_{\mathrm{M}}^{\mathrm{sym}}$ as a Hopf subalgebra. This structure is defined analogously to the one on $Z_{\mathrm{MM}}$ above, replacing each megamatroid by its isomorphism class: e.g. multiplication is given by the direct sum of megamatroids, and is now commutative. The map $\pi_{\mathrm{MM}}$ of (1) is a Hopf algebra morphism.

The space $P_{\mathrm{MM}}$ inherits a Hopf algebra structure from $Z_{\mathrm{MM}}$. Most of this structure can be defined in the expected fashion, but the coproduct requires some care. We define $\Delta: P_{\mathrm{MM}} \rightarrow P_{\mathrm{MM}} \otimes P_{\mathrm{MM}}$ by

$$
\Delta\left(\left[R_{\mathrm{MM}}(\underline{X}, \underline{r})\right]\right)=\sum_{i=0}^{k}\left[R_{\mathrm{MM}}\left(\widehat{\underline{X}_{i}, \underline{r}_{i}}\right)\right] \otimes\left[R_{\mathrm{MM}}\left(\widehat{\underline{X}^{i}, \underline{r}^{i}}\right)\right] .
$$

Since the $R_{\mathrm{MM}}(\underline{X}, \underline{r})$ with $(\underline{X}, \underline{r}) \in \mathfrak{p}_{\mathrm{MM}}=\bigcup_{d, r} \mathfrak{p}_{\mathrm{MM}}(d, r)$ form a basis of $P_{\mathrm{MM}}$, this is sufficient to linearly extend. From Theorem 4.1 one can check that $\left(\Psi_{\mathrm{MM}} \otimes \Psi_{\mathrm{MM}}\right) \otimes \Delta=\Delta \circ \Psi_{\mathrm{MM}}$. 
The Hopf algebra structure on $P_{\mathrm{MM}}$ naturally induces a Hopf algebra structure on $P_{\mathrm{MM}}^{\mathrm{sym}}$ such that $\rho_{\mathrm{MM}}$ and $\Psi_{\mathrm{MM}}^{\mathrm{sym}}$ are Hopf algebra homomorphisms.

\section{Additive valuations}

For $0 \leq e \leq d$ we define $P_{* \mathrm{M}}(d, r, e) \subseteq P_{* \mathrm{M}}(d, r)$ as the span of all $[\Pi]$ where $\Pi \subseteq \mathbb{R}^{d}$ is a $*$ matroid polytope of dimension $\leq d-e$. We have $P_{* \mathrm{M}}(0, r, 0)=P_{* \mathrm{M}}(0, r)$ and $P_{* \mathrm{M}}(d, r, 1)=P_{* \mathrm{M}}(d, r)$ for $d \geq 1$. These subgroups form a filtration

$$
\cdots \subseteq P_{* \mathrm{M}}(d, r, 2) \subseteq P_{* \mathrm{M}}(d, r, 1) \subseteq P_{* \mathrm{M}}(d, r, 0)=P_{* \mathrm{M}}(d, r) .
$$

Define $\bar{P}_{* \mathrm{M}}(d, r, e):=P_{* \mathrm{M}}(d, r, e) / P_{* \mathrm{M}}(d, r, e+1)$, and $T_{\star \mathrm{M}}(d, r)=\bar{P}_{\star \mathrm{M}}(d, r, 1)$. The image of $[Q(M)]$ in $T_{* \mathrm{M}}(d, r)$ is zero if and only if $M$ is connected. The associated graded algebra $\bar{P}_{* \mathrm{M}}=$ $\bigoplus_{d, r, e} \bar{P}_{* \mathrm{M}}(d, r, e)$ has an induced Hopf algebra structure.

Define

$$
\bar{P}_{* \mathrm{M}}(\underline{X})=\bigoplus_{r_{1}, r_{2}, \ldots, r_{e} \in \mathbb{Z}} T_{* \mathrm{M}}\left(\left|X_{1}\right|, r_{1}\right) \otimes \cdots \otimes T_{* \mathrm{M}}\left(\left|X_{e}\right|, r_{e}\right) .
$$

There is a group homomorphism $\bar{\phi}_{\underline{X}}: \bar{P}_{* \mathrm{M}}(\underline{X}) \rightarrow \bar{P}_{* \mathrm{M}}(d, r, e)$. which takes the classes of a list of $*$ matroids to the class of their direct sum. The next theorem essentially asserts a unique decomposition of $*$ matroids into connected components.

Theorem 8.1 We have the isomorphism

$$
\left(\sum_{\underline{X}} \bar{\phi}_{\underline{X}}\right): \bigoplus_{\substack{\underline{X}=\left(X_{1}, X_{2}, \ldots, X_{e}\right) \\ \underline{d}=X_{1} \sqcup X_{2} \sqcup \cdots \sqcup X_{e} ; X_{1}, \ldots, X_{e} \neq \emptyset}} \bar{P}_{* \mathrm{M}}(\underline{X}) \rightarrow \bigoplus_{r \in \mathbb{Z}} \bar{P}_{* \mathrm{M}}(d, r, e)
$$

If $d \geq 1$, let $\mathfrak{t}_{\mathrm{PM}}(d, r)$ be the set of all pairs $(\underline{X}, \underline{r}) \in \mathfrak{p}_{\mathrm{PM}}(d, r)$ such that $r_{1}>0$, and $d \notin X_{k-1}$, where $k$ is the length of $\underline{X}$. Similarly, if $d \geq 2$, let $\mathfrak{t}_{\mathrm{M}}(d, r)$ be the set of all pairs $(\underline{X}, \underline{r}) \in \mathfrak{t}_{\mathrm{M}}(d, r)$ such that $r_{1}>0,\left|X_{k-1}\right|-r_{k-1}<d-r$, and $d \notin X_{k-1}$.

Theorem 8.2 The group $T_{(\mathrm{P}) \mathrm{M}}(d, r)$ is freely generated by all $\left[R_{(\mathrm{P}) \mathrm{M}}(\underline{X}, \underline{r})\right]$ with $(\underline{X}, \underline{r}) \in \mathfrak{t}_{(\mathrm{P}) \mathrm{M}}(d, r)$.

\section{Additive invariants}

The algebra $P_{\star M}^{\text {sym }}$ also has a natural filtration:

$$
\cdots \subseteq P_{\star \mathrm{M}}^{\mathrm{sym}}(d, r, 2) \subseteq P_{\star \mathrm{M}}^{\mathrm{syy}}(d, r, 1) \subseteq P_{\star M}^{\mathrm{sym}}(d, r, 0)=P_{\star \mathrm{M}}^{\mathrm{sym}}(d, r) .
$$

Here $P_{\star \mathrm{M}}^{\mathrm{sym}}(d, r, e)$ is spanned by the indicator functions of all $*$ matroid base polytopes of rank $r$ and dimension $d-e$. Define $\bar{P}_{\star \mathrm{M}}^{\mathrm{sym}}(d, r, e)=P_{* \mathrm{M}}^{\mathrm{sym}}(d, r, e) / P_{* \mathrm{M}}^{\mathrm{sym}}(d, r, e+1)$. Let $\bar{P}_{\star \mathrm{M}}^{\mathrm{sym}}=\bigoplus_{d, r, e} \bar{P}_{\star \mathrm{M}}^{\mathrm{sym}}(d, r, e)$ be the associated graded algebra.

Define $T_{\star \mathrm{M}}^{\mathrm{sym}}=\bigoplus_{d, r} \bar{P}_{\star \mathrm{M}}^{\mathrm{syy}}(d, r, 1)$. The following theorem follows from Theorem 8.1

Theorem 9.1 The algebra $\bar{P}_{\star \mathrm{M}}^{\mathrm{sym}}$ is the free symmetric algebra $S\left(T_{\star \mathrm{M}}^{\mathrm{sym}}\right)$ on $T_{\star \mathrm{M}}^{\mathrm{sym}}$, and there exists an isomorphism

$$
S^{e}\left(T_{\star \mathrm{M}}^{\mathrm{sym}}\right) \cong \bigoplus_{d, r} \bar{P}_{\star \mathrm{M}}^{\mathrm{sym}}(d, r, e) .
$$

Corollary 9.2 The algebra $P_{\star \mathrm{M}}^{\mathrm{sym}}$ is a polynomial ring over $\mathbb{Z}$. 


\section{Invariants as elements in free algebras}

Let

$$
\left(P_{* \mathrm{M}}^{\mathrm{sym}}\right)^{\#}:=\bigoplus_{d, r} P_{* \mathrm{M}}^{\mathrm{sym}}(d, r)^{\vee}
$$

be the graded dual of $P_{* \mathrm{M}}^{\mathrm{sym}}$. Let $\mathfrak{m}_{\star \mathrm{M}}=\bigoplus_{d, r} P_{* \mathrm{M}}^{\mathrm{syy}}(d, r, 1)$. Then we have $\mathfrak{m}_{\star \mathrm{M}}^{2}=\bigoplus_{d, r} P_{* \mathrm{M}}^{\mathrm{sym}}(d, r, 2)$ and $T_{* \mathrm{M}}^{\mathrm{sym}}=\mathfrak{m}_{* \mathrm{M}} / \mathfrak{m}_{* \mathrm{M}}^{2}$. The graded dual $\mathfrak{m}_{\star \mathrm{M}}^{\#}$ can be identified with

$$
\left(P_{\star \mathrm{M}}^{\mathrm{sym}}\right)^{\#} / P_{* \mathrm{M}}^{\mathrm{sym}}(0,0) \cong \bigoplus_{d=1}^{\infty} \bigoplus_{r} P_{\star \mathrm{M}}^{\mathrm{sym}}(d, r)^{\vee} .
$$

So $\mathfrak{m}_{\mathrm{PM}}^{\#} \otimes_{\mathbb{Z}} \mathbb{Q}$ will be identified with the ideal $\left(u_{0}, u_{1}, \ldots\right)$ of $\mathbb{Q}\left\langle u_{0}, u_{1}, \ldots\right\rangle$ and $\mathfrak{m}_{\mathrm{M}}^{\#} \otimes_{\mathbb{Z}} \mathbb{Q}$ will be identified with the ideal $\left(u_{0}, u_{1}\right)$ of $\mathbb{Q}\left\langle u_{0}, u_{1}\right\rangle$. The graded dual $\left(T_{\mathrm{PM}}^{\mathrm{sym}}\right)^{\#} \otimes_{\mathbb{Z}} \mathbb{Q}$ is a subalgebra (without 1) of the ideal $\left(u_{0}, u_{1}, \ldots\right)$, and $\left(T_{\mathrm{PM}}^{\mathrm{sym}}\right)^{\#} \otimes_{\mathbb{Z}} \mathbb{Q}$ is a subalgebra of $\left(u_{0}, u_{1}\right)$.

Theorem 10.1 Let $u_{0}, u_{1}, u_{2}, \ldots$ be indeterminates, where $u_{i}$ has bidgree $(1, i)$. We have the following isomorphisms of bigraded associative algebras over $\mathbb{Q}$ :

a. The space $\left(P_{M}^{\mathrm{sym}}\right)^{\vee} \otimes_{\mathbb{Z}} \mathbb{Q}$ is isomorphic to $\mathbb{Q}\left\langle\left\langle u_{0}, u_{1}\right\rangle\right\rangle$, the completion (in power series) of the free associative algebra generated by $u_{0}, u_{1}$.

b. The space $\left(P_{\mathrm{PM}}^{\mathrm{sym}}\right)^{\vee} \otimes_{\mathbb{Z}} \mathbb{Q}$ is isomorphic to $\mathbb{Q}\left\langle\left\langle u_{0}, u_{1}, u_{2}, \ldots\right\rangle\right\rangle$.

c. The space $\left(T_{\mathrm{M}}^{\mathrm{sym}}\right)^{\vee} \otimes_{\mathbb{Z}} \mathbb{Q}$ is isomorphic to $\mathbb{Q}\left\{\left\{u_{0}, u_{1}\right\}\right.$, the completion of the free Lie algebra generated by $u_{0}, u_{1}$.

d. The space $\left(T_{\mathrm{PM}}^{\mathrm{sym}}\right)^{\vee} \otimes_{\mathbb{Z}} \mathbb{Q}$ is isomorphic to $\mathbb{Q}\left\{\left\{u_{0}, u_{1}, u_{2}, \ldots\right\}\right.$.

Proposition 10.2 The Hopf algebra $P_{\mathrm{PM}}^{\mathrm{sym}} \otimes_{\mathbb{Z}} \mathbb{Q}$ is isomorphic to the ring QSym of quasi-symmetric functions over $\mathbb{Q}$.

If we identify $P_{\mathrm{PM}}^{\mathrm{sym}} \otimes_{\mathbb{Z}} \mathbb{Q}$ with $Q S y m$, then $\mathcal{G}$ is equal to $\Psi_{\mathrm{PM}}^{\mathrm{sym}}$.

\section{Acknowledgements}

The second author is grateful to David Speyer for collaboration which led to a proof of Theorem 1.4 before he got in touch with the first author.

\section{References}

[1] F. Ardila, A. Fink, F. Rincón, Valuations for matroid polytope subdivisions, Canadian Math. Bulletin 13, to appear.

[2] A. Björner, M. Las Vergnas, B. Sturmfels, N. White, and G. Ziegler. Oriented Matroids. Encyclopedia of Mathematics and Its Applications, vol. 46. Cambridge University Press, Cambridge, 1993.

[3] L.J. Billera, N. Jia, V. Reiner, A quasi-symmetric function for polymatroids, to appear in the European Journal of Combinatorics, arXiv:math/0606646 
[4] C. J. Brianchon, Théorème nouveau sur les polyèdres convexes, J. École Polytechnique 15 (1837), 317-319.

[5] T. H. Brilawski, The Tutte-Grothendieck ring, Algebra Universalis 2 (1972), 375-388.

[6] H. Crapo, Single-element extensions of matroids, J. Res. Nat. Bur. Standards Sect. B 69B (1965), $55-65$.

[7] H. Crapo, The Tutte polynomial, Aequationes Math. 3 (1969), 211-229.

[8] H. Derksen, Symmetric and quasi-symmetric functions associated to polymatroids, preprint, arXiv: 0801.4393

[9] J. Edmonds, Submodular functions, matroids, and certain polyhedra, in: R. Guy, H. Hanani, N. Sauer, J. Schonheim (eds.), Combinatorial Structures and Their Applications, Gordon and Breach, New York, 1970, 69-87.

[10] I. M. Gel'fand, M. Goresky, R. MacPherson, V. Serganova, Combinatorial geometries, convex polyhedra and Schubert cells, Adv. in Math. 63 (1987), 301-316.

[11] J. P. Gram, Om rumvinklerne i et polyeder, Tidsskrift for Math. (Copenhagen) (3) 4 (1874), 161-163.

[12] H. Groemer, On the extension of additive functionals on classes of convex sets, Pacific J. Math. 75 (1978), no. 2, 397-410.

[13] J. Herzog, T. Hibi, Discrete polymatroids, J. Algebraic Combinatorics 16 (2002), no. 2, 239-268.

[14] L. Lafforgue, Pavages des simplexes, schémas de graphes recollés et compactification des $\mathrm{PGL}_{r}^{n+1} / \mathrm{PGL}_{r}$, Invent. Math. 136 (1999), no. 1, 233-271.

[15] L. Lafforgue, Chirurgie des Grassmanniennes, CRM Monograph Series 19, AMS, Providence, RI, 2003.

[16] J. Morton, L. Pachter, A. Shiu, B. Sturmfels, O. Wienand, Convex rank tests and semigraphoids, arXiv:math/0702564v2.

[17] J. G. Oxley, Matroid theory, Oxford University Press, New York, 1992.

[18] A. Postnokiv, Permutohedra, associahedra, and beyond, Int. Math. Res. Notices (2009), doi:10.1093/imrn/rnn153.

[19] D. Speyer, A matroid invariant via the K-theory of the Grassmannian, to appear in Advances in Math., arXiv:math/0603551.

[20] W. Volland, Ein Fortsetzungssatz für additive Eipolyederfunktionale im euklidischen Raum, Arch. Math. 8 (1957), 144-149.

[21] D. J. A. Welch, Matroid theory, London Mathematical Society Monographs 8, Academic Press, London, New York, 1976.

[22] H. Whitney, On the abstract properties of linear independence, Amer. J. of Math. 57 (1935), 509533. 